\section{MS15-P27 Prediction of isostructural series of solvates}

Iva Koupilová ${ }^{1}$, Michal Hušák ${ }^{1}$, Jan Rohlíček ${ }^{1}$

1. Department of solid state chemistry, UCT Prague, Prague, Czech Republic

\section{email: iva.koupilova@vscht.cz}

Organic molecules can usually form crystal solvated structures with a multiple different solvents. In general, there are two possible situations how the solvate can be formed: (i) the solvate creates a new crystal structure in a new structural type by changing the space group and/or lattice parameters, or (ii) the solvate creates a crystal structure in the same space group and similar unit cell where only the molecule of solvent is changed. We call this second case as a series of isostructural solvates. Bromocriptine mesylate (BCM) forms two series of isostructural solvates. 21 new solvates of $\mathrm{BCM}$ were prepared in the space group $P 2$ and three more solvates were prepared in the space group $P 22_{1} 2_{1}$. In BCM's case, we found a correlation between the volume of the unit cell and the volume of the solvent's molecule. We inspired by this BCM case and we have created a general algorithm to identify more isostructural series of solvates from the Cambridge structural database (CSD). The algorithm works on the basis of similarity evaluation by comparing reduced unit cell parameters, reduced unit cell volume and space group of actualized list of solvates' groups from work van der Streek ${ }^{1}$. The aim of this work is to confirm correlation between the change of the volume of the unit cell and the volume of the solvent's molecule and also to find a predictive model of new series of isostructural solvates.

Financial support from specific university research: MSMT No 20/2015. van de Streek, J. Crystengcomm 2007, 9, 350.

Keywords: isostructural solvates, prediction, bromocriptine mesylate

\section{MS15-P28 Using time-resolved X-ray diffraction to test the piezoelectricity of the field-stabilized polar phase in $\mathrm{SrTiO}_{3}$ \\ Behnam Khanbabaee ${ }^{1}$, Carsten Richter ${ }^{2}$, Erik Mehner ${ }^{2}$, Juliane} Hanzig $^{2}$, Semen Gorfman ${ }^{1}$, Dirk C. Meyer ${ }^{2}$, Ullrich Pietsch ${ }^{1}$

1. Solid State Physics, Department of Physics, University of Siegen, Germany

2. Institute of Experimental Physics TU Bergakademie Freiberg, Germany

email: khanbabaee@physik.uni-siegen.de

The aim of this contribution is to test the piezoelectricity of a $\mathrm{SrTiO}_{3}$ single crystal using stroboscopic time-resolved X-ray diffraction. Although the cubic perovskite and, hence, centrosymmetric structure of $\mathrm{SrTiO}_{3}$ at room temperature prohibits the appearance of piezoelectricity, it is known that application of a static electric field for extended period of time ( 12 hours) breaks this symmetry and creates the migration induced field stabilized polar (MFP) phase [1]. In the present work, we used time-resolved X-ray diffraction to test piezoelectricity of this phase. We started the measurements by applying a static voltage of $\mathrm{U}_{0}=100 \mathrm{~V}$ (corresponding to the electric field of $1 \mathrm{kV} / \mathrm{mm}$ ) for around $12 \mathrm{~h}$ in order to form the MFP phase followed by changing the voltage to dynamical mode by adding a time dependent, triangular shaped AC component to the above mentioned static voltage. Then, various symmetrical and asymmetrical Bragg reflections of SrTiO3 were scanned under applied $1 \mathrm{kHZ}$ triangular shape electric field. The results for the 002 reflection (see Fig. 1) clearly show the electric field induced angular shift of the Bragg peak corresponding to the MFP phase, while the position of the bulk peak was always fixed. This result indicated that the MFP is indeed piezoelectric. The variation of the Bragg position with applied periodic electric field allows estimating the piezoelectric coefficient $\left(\mathrm{d}_{33}\right)$, describing the longitudinal elongation in response to field applied along the crystallographic $c$ axis (polar axis of the MFP phase). From the observed shifts of the 002 reflection, we estimated that the $d_{33}>27.3$ $\mathrm{pC} / \mathrm{N}$ which is $\sim 10$ higher than e.g. such of quartz.

[1] J. Hanzig, M. Zschornak, et al., Phys. Rev. B 88, 024104 (2013), doi:10.1103/PhysRevB.88.024104 


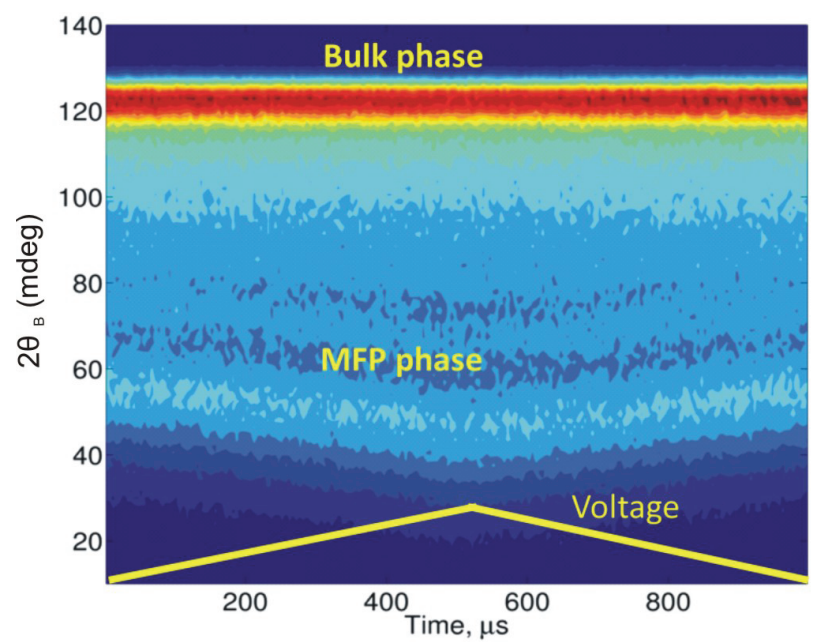

Figure 1. Time resolved X-ray diffraction 002 reflection under triangular shape $1 \mathrm{kHZ}$ electric field (yellow curve). The top sharp peak corresponds to the bulk and deviated weak bottom peak corresponds to MFP phase

Keywords: time-resolved X-ray diffraction, SrTiO3, piezoelectricity

\section{MS15-P29 Synthesis, crystal structure, spectroscopic characterization and theoretical study of Nicotinaldehyde $\mathrm{N}$-phenylsemicarbazone}

Rafael Mendoza-Meroño ${ }^{1}$, Rubén Soria-Martínez ${ }^{1}$, Santiago García-Granda $^{1}$

1. Facultad de Química.Departamento de Química-Física y Analítica. Universidad de Oviedo

\section{email: rafam80@gmail.com}

Semicarbazones are derivative of imines compounds, which could be obtained by the condensation reaction between a ketones or aldehydes and semicarbazides. Semicarbazones exist in two tautomeric forms, keto (A) and enol (B) forms. The keto form acts as bidentate neutral ligand and the enol form can deprotonates acting as anionic ligand, making the semicarbazones versatile ligands in both neutral and anionic forms $[1,2]$. The coordination mode is influenced by the number and type of substituents groups; this is because the active donor sites of the ligand vary depending upon the substituents. In view of the importance of these compounds, the synthesis, crystal structure, spectroscopic characterization and theoretical study of Nicotinaldehyde $\mathrm{N}$-phenylsemicarbazone have been carried out. In the first part of this study the semicarbazone molecule was synthesized and characterized by FT-IR, FT-Raman and NMR. The crystal structure was determined by X-ray single-crystal diffraction. The molecules crystallize in a $\mathrm{P} 2 / \mathrm{c}$ space group and form in the crystal packing $\mathrm{N}-\mathrm{H} . . \mathrm{O}$ and $\mathrm{N}-\mathrm{H} . . . \mathrm{N}$ hydrogen bonds forming a centrosymmetric synthon. Other interactions like $\mathrm{C}-\mathrm{H} \ldots \pi$ and $\pi \ldots \pi$ stacking helps to stabilized the crystals. In the second part, the molecular geometry was optimized using DFT method and compared with the experimental data obtained from X-ray single-crystal experiment. The experimental (FT-IR) and calculated vibrational frequencies (using DFT) have been compared. The stability and charge delocalization was studied by natural bond orbital (NBO) analysis as well as the potential energy distribution (PED). Milliken population analysis on atomic charges is also calculated.

Acknowledments: Spanish Ministerio de Economía y Competitividad (MAT2013-40950-R) and ERDF funding is acknowledged.

[1] R.Pavan, P. Maia, S.Leite, S. Deflon, V. Batista, A. (2010). Eur J Med Chem. 2010 45, 1898-1905. [2]P.Yogeeswari,P.,D. Sriram , V. Veena,, R.Kavya, R., K.Rakhra., J.Ragavendran, S.Mehta, R.Thirumurugan, Biomedicine \& Pharmacotherapy. 2005 59, 51-55.

Keywords: Semicarbazone, single-crystal X-ray diffraction, DFT studies. 